This item was submitted to Loughborough's Research Repository by the author.

Items in Figshare are protected by copyright, with all rights reserved, unless otherwise indicated.

\title{
Time to act: the challenges of working during and after cancer, initiatives in research and practice
}

\section{PLEASE CITE THE PUBLISHED VERSION}

http://dx.doi.org/10.1016/j.ejon.2014.01.001

\section{PUBLISHER}

(c) ELSEVIER

\section{VERSION}

AM (Accepted Manuscript)

\section{PUBLISHER STATEMENT}

This work is made available according to the conditions of the Creative Commons Attribution-NonCommercialNoDerivatives 4.0 International (CC BY-NC-ND 4.0) licence. Full details of this licence are available at: https://creativecommons.org/licenses/by-nc-nd/4.0/

\section{LICENCE}

CC BY-NC-ND 4.0

\section{REPOSITORY RECORD}

Wells, Mary, Ziv Amir, Tom Cox, Gail Eva, Diana Greenfield, Gill Hubbard, Richard Kyle, et al.. 2019. "Time to Act: The Challenges of Working During and After Cancer, Initiatives in Research and Practice”. figshare. https://hdl.handle.net/2134/17200. 


\section{EJON Editorial}

Accepted version

\section{Time to act: The challenges of working during and after cancer, initiatives in research and practice}

Mary Wells, Ziv Amir, Tom Cox, Gail Eva, Diana Greenfield, Gill Hubbard, Richard Kyle, Sara McLennan, Fehmidah Munir, Sarah Scott, Linda Sharp, Tyna Taskila, Theresa Wiseman (the CANWORK research group)

Published in: European Journal of Oncology Nursing 18 (2014) 1-2.

A diagnosis of cancer has a significant impact on work and employment. With improvements in cancer treatments more people are surviving longer, and it is estimated that there approximately 700,000 people of working age with cancer in the UK [1]. This figure is increasing year on year [2], not least because people are working later in life. While many people are able to remain in or return to work, a sizeable number experience problems: research shows that people with cancer are 1.37 times more likely to be unemployed than those without [3]. Patients experience a lack of well-timed and appropriate support, both in the acute phase and in the months and years following treatment. Health care professionals, in turn, report that they feel ill-equipped to respond adequately to patients' work-related difficulties [4].

As well as providing much needed financial stability, the ability to work is important to maintaining social relationships, self-esteem and psychological well-being. A recent systematic review and metasynthesis of the qualitative literature on employment and cancer [5] found consistent evidence that individuals' experiences of 'return to work' were strongly influenced by the degree to which cancer affected four key factors: self-identity, meaning and significance of work, family and financial context, and work environment. Work often helped people to re-establish normality and a sense of their former selves, but at the same time, self-identity was challenged by the physical and emotional consequences of cancer treatment, as well as others' reactions to changes in appearance, physical or cognitive ability. The meaning and significance that individuals had attached to work before cancer frequently shifted, as they re-evaluated their capabilities and priorities. The influence of financial pressures and family or friends towards maintaining or changing work consistently played a part, and the difficulties of negotiating sick pay, benefits and insurance were frequently mentioned. Finally, the organisational and interpersonal support received within the work environment played a particularly important role in enabling people to talk about and manage the challenges they faced, negotiate different work patterns and responsibilities and regain confidence at work.

A recent cost-analysis across the European Union estimated that $60 \%$ of the economic burden of cancer was incurred in non-health-care areas, with almost $€ 43$ billion in lost productivity attributable to early death, and $€ 83$ million lost working days due to cancer related morbidity [6]. Welfare legislation in many western countries makes it the legal responsibility of the employer to make 'reasonable adjustments' to allow continuing participation in employment for people with disability including cancer. Yet a large proportion of those completing cancer treatment return to work only to leave within the year, reporting reintegration as too difficult. 
Cancer survivors identify the role played by employers as important in supporting and sustaining a successful return to work and in supporting career development. There is an increasing trend towards the devolution of human resource (HR) work to line managers. This devolvement means that line managers' role in the return to work process following illness is likely to be central. However, a UK study [7] of line managers' experiences suggests that employers/ line-managers need to be provided with training, support, and resources to help them facilitate employment and job retention of employees diagnosed with cancer. Furthermore, most respondents in a UK national survey of Occupational Health $(\mathrm{OH})$ physicians [4] felt managers treated referral to $\mathrm{OH}$ differently for employees with cancer compared to management referral for employees with other diagnoses.

Unfortunately, many small and medium sized enterprises (and some large enterprises) lack the necessary resources to adequately support the health and well-being of their employees. In response to this, practical tools have been developed in the UK to help line managers support an employee diagnosed with cancer throughout their cancer journey in relation to work [8-11]. This includes support for an employee taking sick leave and returning to work, or an employee who chooses to continue to work during treatment. The Line Manager Behaviour Checklist $[8,9]$ was developed for use with a range of chronic conditions, including cancer, and can be accessed by line managers through the Chartered Institute of Personnel Development (CIPD). A specific resource for those diagnosed with cancer is the 'Work it Out' tool [11], part of a Toolkit for employees, employers and health professionals, available from the UK Charity Macmillan Cancer Support. This tool allows the employee to feel in control of decision-making by requesting appropriate information and support not just from their line manager, but also from other relevant stakeholders such as human resources and their oncology nurse or consultant $[10,11]$. Although such tools are valuable in minimising some of the undesirable outcomes of work issues for those diagnosed with cancer, the current literature suggests that much more needs to be done to enable those affected by cancer to sustain employment.

Recognising the growing interest and activity in research focussed on cancer and work in the UK and Ireland, a multidisciplinary group of researchers met at the University of Dundee in May 2011 to share ideas and develop collaborations. The CanWork research network was subsequently established, aiming to drive forward a programme of research on cancer, employment and finance [12]. More recently, the European Cooperation in Science and Technology (COST) has funded CANWON - a network of expert scientists, clinicians, economists and patient support groups and other stakeholders on cancer and work [13]. COST is an intergovernmental framework allowing the coordination of nationally-funded research on European level; it does not fund research itself, but it provides support for networking activities and facilitates disseminating research knowledge and best practice worldwide. The CANWON network currently connects 24 researchers from $15 \mathrm{EU}$ countries. The network operates through four work groups: (1) prognostic factors of unemployment in cancer survivors including gender- and country-specific differences; (2) work-related costs of survivorship for both patients and society; (3) the role of employers; and (4) development and evaluation of innovative, interdisciplinary interventions which effectively support employment. The network will make earlier and quicker exchange of new information possible. It also aims to increase the impact of research on cancer and work among policy makers, regulatory bodies and national decision makers as well as the private sector. 
These initiatives aim to raise awareness of how cancer affects working life, and to draw attention to the need for more research into neglected areas such as: the needs and experiences of particular groups e.g. the self-employed; the views and experiences of line-managers working in small and medium enterprises (SME); the nature of the advice and guidance required by line-managers; potential ways to improve the communication between line-managers, their senior management and relevant occupational health advisers and the development and evaluation of effective interventions to support the achievement of work-related goals in cancer survivors.

Nurses and other practitioners working in cancer care also need to recognise the importance of work and take opportunities to assess and consider how cancer and its treatment may interfere with a person's ability to maintain their working life, and as a result, their identity and financial security. Implementing work-related information, advice and support into clinical practice is an important next step. Front line oncology staff need not be experts in this field but need to be prepared to open this dialogue from the outset, asking appropriate questions, advising on the most likely course of events and how these may impact on work. In order to manage expectations and help individuals plan for their future working life, written and verbal information and support should be provided throughout the cancer pathway, particularly around the time of diagnosis and at the end of treatment. Oncology nurses have a key role to play in signposting to further advice and support, for example to hospital-based social workers (where they exist), vocational rehabilitation services, local cancer support and information centres. Perhaps most importantly, nurses are in a position to ensure that an understanding of the complexities of work-related decisions and experiences becomes integral to the care of people with cancer.

References:

1. Maddams J, Brewster D, Gavin A, Steward J, Elliott J, Utley M, Møller H (2009) Cancer prevalence in the United Kingdom: estimates for 2008. British Journal of Cancer 101: 541 547.

2. Maher J, McConnell H (2009) New pathways of care for cancer survivors: adding the numbers British Journal of Cancer 105, S5-S10

3. de Boer AG, Taskila T, Ojajarvi A, van Dijk FJ, Verbeek JH (2009) Cancer survivors and unemployment: a meta-analysis and meta-regression. JAMA 301: 753-762.

4. Amir Z, Wynn P, Whitaker S, and Luker K, (2009), Cancer survivorship and return to workUK Occupational Health Physician experiences, Occupational Medicine, 59(6), 390-396

5. Wells $\mathrm{M}_{\mathbf{L}}$ Williams B, Firnigl D, MacGillivray S, Lang H, Coyle J, Kroll T Supporting "workrelated goals" rather than "return to work" after cancer? A systematic review and metasynthesis of 25 qualitative studies PsychoOncology. 2012 Aug 10. doi: 10.1002/pon.3148

6. Luengo-Fernandez R, Leal J, Gray A, Sullivan R Economic burden of cancer across the European Union: a population-based cost analysis Lancet Oncology 2013 http://dx.doi.org/10.1016/S1470-2045(13)70442-X

7. Amir Z, Wynn P, Fong C, Strauser D, Whitaker S, Luker K, (2010), Return to work after cancer in the UK: Attitudes and experiences of line managers, Journal of Occupational Rehabilitation, (20), 435-442

8. Yarker J, Munir F, Donaldson-Feilder E, Hicks B.(2010) Managing rehabilitation: Competency framework for managers to support return to work. London: BOHRF. 
9. Munir, F., Yarker, J., Hicks, B., \& Donaldson-Feilder, E. (2012). Returning employees back to work: Developing a measure for Supervisors to Support Return to Work (SSRW). Journal of Occupational Rehabilitation, 22, 196-208.

10. Munir, F., Kalawsky, K., Wallis D., \& Donaldson-Feilder, E. (2013). Using intervention mapping to develop a work-related guidance tool for those affected by cancer. BMC Public Health, 13, 6. DOI: 10.1186/1471-2458-13-6. URL: http://www.biomedcentral.com/14712458/13/6

11. Munir, F., Wallis, D., Kalawsky., K., Donaldson-Feilder, E. (2011). Work it out: The essential questions to ask about work. London: Macmillan Cancer Support (available from http://www.macmillan.org.uk/Documents/GetInvolved/Campaigns/WorkingThroughCancer LWorkltOut/WorkltOut.pdf - accessed 9/12/13)

12. CanWork: Establishing a UK/Ireland network for research on cancer and employment. Poster presentation at NCRI conference $2011 \mathrm{http}$ ://www.richardgkyle.com/wpcontent/uploads/2011/10/NCRI-CanWork-Poster.pdf

13. De Boer A The European Cancer and Work Network CANWON. Journal of Occupational Rehabilitation. Vol. 23 (3). September 2013.

\section{CANWORK group members (in alphabetical order and by institution)}

Prof Ziv Amir

Dr Gail Eva, NIHR Post-doctoral Research Fellow, UCL Institute of Neurology, Queen Square London WC1N 3BG

Dr Diana Greenfield

Dr Gill Hubbard and Dr Richard Kyle, Cancer Care Research Centre, School of Nursing, Midwifery and Health, University of Stirling, Centre for Health Science, Old Perth Road, Inverness IV2 3JH

Dr Fehmidah Munir, School of Sport, Exercise and Health Sciences, Loughborough University, Loughborough, Leicestershire LE11 3TU

Dr Sarah Scott, Prof Tom Cox and Dr Sara McLellan, Academic Urology Unit, Health Sciences Building (2nd Floor), University of Aberdeen, Foresterhill, Aberdeen AB25 2ZD

Dr Linda Sharp

Dr. Tyna Taskila, Senior Researcher, The Work Foundation, Centre for Workforce Effectiveness 21 Palmer Street, London SW1H OAD

Prof. Mary Wells, Professor of Cancer Nursing Research \& Practice, NMAHP Research Unit, University of Stirling Stirling FK9 4LA

Dr Theresa Wiseman 